\section{Associação entre dor crônica e autorrelato de quedas: estudo populacional - SABE}

\author{
Association between chronic pain and \\ self-reported falls in the SABE study population
}

\author{
Asociación entre dolor crónico y auto-reporte \\ de las caídas: estudio poblacional - SABE
}

\author{
${ }^{1}$ Universidade Estadual de \\ Londrina, Londrina, Brasil. \\ 2 Escola de Enfermagem, \\ Universidade de São Paulo, \\ São Paulo, Brasil. \\ 3 Faculdade de Saúde \\ Pública, Universidade de São \\ Paulo, São Paulo, Brasil. \\ Correspondência \\ M. S. G. Dellaroza \\ Universidade Estadual de \\ Londrina. \\ Rua Borba Gato 1078, apto \\ 904, Londrina, PR 86010-630 \\ Brasil. \\ dellaroza@uel.br
}

\begin{abstract}
The objective of this study was to assess the association between chronic pain and self-reported falls. This was a cross-sectional sample of elderly individuals without cognitive deficits, living in the city of São Paulo, Brazil, and with chronic pain. The study considered elderly that reported chronic pain for at least one year. History of falls was defined as at least one reported fall in the 12 months prior to the study. Data were obtained by home surveys of the elderly. Stata 11.0 was used for statistical analysis. Prevalence of chronic pain was 29.7\% (95\%CI: 25.4-33.9). Prevalence of falls in the previous year for individuals with pain was 31.6\% (95\%CI: 26.4-37.5) and did not differ significantly from those without pain (26.4\%; 95\%CI: 23.1-30.0; $p=0.145)$. Risk of falls was 50\% higher $(p=0.019)$ for those with pain and osteoporosis and 48\% higher for those with pain and urinary incontinence $(p=0.010)$. History of pain for at least one year and osteoporosis, pain, and urinary incontinency showed higher odds of falls.
\end{abstract}

Aged; Pain; Accidental Falls

\author{
Mara Solange Gomes Dellaroza ${ }^{1}$ \\ Cibele Andrucioli de Mattos Pimenta 2 \\ Maria Lúcia Lebrão ${ }^{3}$ \\ Yeda Aparecida de Oliveira Duarte 2 \\ Patricia Emília Braga 2
}

\section{Resumo}

Estudo populacional transversal com idosos sem déficit cognitivo residentes na cidade de São Paulo, Brasil. O objetivo foi avaliar a associação entre dor crônica e autorrelato de quedas. Idoso com dor crônica foi considerado aquele com dor de duração há mais de um ano. Os dados foram coletados por entrevista domiciliar, e as análises foram realizadas no programa Stata 11.0. A prevalência de dor crônica foi 29,7\% (IC95\%: 25,433,9); a prevalência de queda, no último ano, en tre idosos com dor foi 31,6\% (IC95\%: 26,4-37,5) $e$ não diferiu da prevalência entre idosos sem dor (26,4\%; IC95\%: 23,1-30,0; $p=0,145)$. No entanto, quando havia dor e osteoporose ou dor e incontinência urinária, o risco de quedas foi $50 \%$ ( $p=$ $0,019)$ e 48\% maior ( $p=0,010$ ), respectivamente. A associação dor crônica e osteoporose ou incontinência urinária elevou a chance de quedas em idosos.

Idoso; Dor; Acidentes por Quedas 


\section{Introdução}

Frequentes entre idosos, quedas têm prevalência e incidência que variam conforme a faixa etária e o delineamento da pesquisa. Estudo com idosos residentes na comunidade em São Paulo, Brasil, revelou incidência de $31 \%$ de quedas e $11 \%$ de duas ou mais quedas 1 .

As principais consequências da queda são o declínio de condições físicas e mentais, com aumento do risco de outras quedas, além da piora da qualidade de vida relacionada à dimensão saúde 2 e o medo de cair novamente. A queda está entre as principais causas de incapacidade e dependência em idosos 3,4, a causa direta de fraturas 2,5 .

Dados do Centro de Vigilância Epidemiológica da Secretária de Saúde do Estado de São Paulo demonstraram que o coeficiente de mortalidade por quedas foi de 31/100 mil habitantes, chegando a 110,7/100 mil habitantes na faixa etária de oitenta anos e mais. As internações por quedas, em 2008, foram de 20.726, o que representa $60,7 \%$ do total de internações por causas externas no estado. As quedas do mesmo nível representaram importante parcela de mortes definidas (35\%), internações $(47,5 \%)$ e atendimentos de emergências (66\%), havendo aumento com o avanço da idade 6 .

Diante de tão graves consequências, identificar as causas ou os fatores preditores de quedas é essencial para a prevenção deste agravo e de suas sequelas. Entre as causas relacionadas às quedas, existem fatores intrínsecos e extrínsecos. Estudo de revisão de literatura, que incluiu 15 estudos de coorte prospectivos com, no mínimo, cem idosos, apresentou os seguintes fatores de risco mais frequentes para queda: a alteração de marcha, a debilidade muscular e a incapacidade para as atividades básicas de vida diária (ABVD) e para as atividades instrumentais de vida diária (AIVD) 7 . A associação entre dor crônica e quedas é ainda controversa.

Considera-se dor crônica aquela que persiste por meses ou anos, que pode durar além do tempo de cura de uma lesão, estando ou não associada a doenças crônicas. A Associação Internacional para Estudo da Dor propõe a duração de três meses para caracterizar dor crônica para fins clínicos e seis meses para fins de pesquisa, mas isso nem sempre é seguido e tem sido causa de fragilidade na comparação entre os estudos. A prevalência de dor entre idosos no Brasil e no mundo varia de $37 \%$ a $70 \%$, e essa variabilidade depende do critério de dor crônica, da idade e procedência da população, dos locais de dor e dos delineamentos dos estudos, entre outros 8,9,10.
Em revisão de literatura que envolveu as bases de dados PubMed, LILACS e SciELO, sem limite no período de busca, e combinação dos descritores pain, accidental falls, aged or elderly, encontramos 11 estudos que analisaram a ocorrência de dor e quedas em idosos. Em cinco dessas investigações, verificou-se associação entre a intensidade da dor e a ocorrência de quedas $3,4,10,11,12$. Os locais de dor que mais se associaram ao maior risco de quedas foram pés $3,11 \mathrm{e}$ articulações 12,13. A dor intensa aumentou o risco de queda, conforme um dos estudos 12, e a dor de moderada a intensa apresentou relação no desempenho do equilíbrio e da dinâmica de caminhada 10 . O uso de analgésico diário foi fator de proteção em um estudo 3 .

Apesar de haver indícios de que dor e queda estão associadas, não foi encontrado nenhum estudo com idosos brasileiros que avaliasse essa associação. A prevenção de quedas é prioridade na atenção ao idoso no Brasil, e identificar todos os fatores de risco envolvidos nesse agravo é primordial para que ações preventivas sejam propostas. Portanto, uma vez que ainda não há resposta satisfatória para a questão "Quais características da dor crônica, como local, intensidade e frequência, estão associadas a maior risco de quedas?", justifica-se a elaboração da presente pesquisa, cujos objetivos foram identificar a prevalência de relato de queda e avaliar a associação do relato de queda em idosos com dor crônica com as características da dor, a interferência da dor no trabalho, variáveis sociodemográficas, morbidade autorreferida, funcionalidade e alterações da mobilidade.

\section{Método}

\section{Estudo epidemiológico transversal, com amostra de base populacional}

Os dados foram coletados no banco de dados do projeto SABE - Saúde, Bem-estar e Envelhecimento, um estudo longitudinal planejado para identificar as condições de vida e de saúde de idosos de sete cidades da América Latina e Caribe. Na presente pesquisa, foram analisados os dados coletados no ano de 2006, através de entrevista com idosos residentes na cidade de São Paulo, sem incluir idosos institucionalizados. Nesta pesquisa usaremos o termo "idosos da comunidade" referindo-se a idosos não institucionalizados ou seja que residem em moradias em bairros e vilas da cidade. Naquele ano, foram entrevistados 1.413 idosos. Os critérios de inclusão no estudo foram ter sessenta anos ou mais, sentir dor há mais de um ano ou não sentir dor, apresentar desempe- 
nho igual ou superior a treze (13) pontos no Mini Exame do Estado Mental (MEEM) e igual ou superior a seis (6) no Pfeffer Functional Activities Questionnaire (QFAF), pois esses idosos, devido as boas condições cognitivas, responderam sozinhos ao questionário ou somente com a ajuda de uma pessoa auxiliar. Os critérios de exclusão foram ter informante substituto e sentir dor há menos de um ano.

Assim, foram excluídos 142 indivíduos que tiveram informante substituto e queixa de dor há menos de um ano, além de mais dois idosos que não responderam as questões referentes à ocorrência de queda. A amostra resultou em 1.269 idosos, sendo 340 com dor há pelo menos um ano e 829 sem dor, o que corresponde ao desenho amostral de 895,762 idosos.

A opção por excluir os idosos cujas informações foram dadas por informante substituto deveu-se ao entendimento de que o melhor informante sobre sua dor é quem a sente. Decidiuse incluir na análise apenas os idosos que apresentavam dor há um ano ou mais, visto que o desfecho de autorrelato de queda foi averiguado no último ano que antecedeu a entrevista.

As variáveis independentes analisadas foram características sociodemográficas, como sexo, faixa etária, situação de trabalho e moradia e renda familiar mensal; características da dor crônica que mais incomodava, como localização, duração, intensidade e frequência; ocorrência de sintomas depressivos e autorrelato de morbidade.

A presença de sintomas depressivos foi avaliada pela Escala de Depressão Geriátrica (GDS), de 15 itens. A existência de outras doenças foi verificada pela questão: "Alguma vez um médico ou enfermeiro disse que o Sr. tem... ?”; os idosos que responderam "sim" foram considerados portadores dessas patologias.

A capacidade funcional foi avaliada pelo grau de dependência em atividades básicas e instrumentais de vida diária, e a mobilidade foi mensurada por meio do autorrelato de dificuldade para caminhar a distância de uma rua (quadra), ficar sentado(a) durante duas horas, levantar-se de uma cadeira, subir um andar pelas escadas sem descansar, curvar-se, ajoelhar-se ou agachar-se e puxar ou empurrar grandes objetos.

A interferência da dor no trabalho foi avaliada com base na Escala SF 12 de Qualidade de Vida, por meio da questão: "Durante as últimas quatro semanas (último mês), quanto a presença de dor interferiu no seu trabalho normal?".

Os dados foram inseridos e analisados no banco de dados do programa estatístico Stata 11.0 (Stata Corp., College Station, Estados Unidos). Calcularam-se as prevalências de presença de dor crônica e ocorrência de quedas, com intervalos de 95\% de confiança (IC95\%). Para analisar a relação entre relato de queda e as variáveis independentes, foi utilizado o teste de associação de RaoScott, que considera o desenho amostral. As variáveis que apresentaram valor de $\mathrm{p}$ inferior a 0,25 foram incluídas na análise múltipla. Nesta fase, compararam-se as razões de prevalência obtidas pela regressão múltipla de Cox (com variância robusta). As variáveis que apresentaram valor de $\mathrm{p}<0,05$ ficaram no modelo final.

$\mathrm{O}$ projeto SABE recebeu a aprovação do Comitê de Ética em Pesquisa da Faculdade de Saúde Pública da Universidade de São Paulo, conforme ofício COEP/83/06.

\section{Resultados}

A população pesquisada no projeto SABE 2006, na maior parte dos casos, compôs-se de mulheres $(59,6 \%)$, idosos que não trabalhavam $(68,3 \%)$ e viviam com outras pessoas $(86,6 \%)$. A idade mais frequente variou entre 65 e 74 anos $(48,4 \%)$; $21,9 \%$ tinham mais de 75 anos, e a média de idade foi de 69,5 anos (erro padrão de 0,6). A escolaridade ficou entre um e quatro anos $(59,4 \%)$, com média de anos de escolaridade de 4,3 (erro padrão de 0,2). A renda familiar foi de até três salários mínimos, segundo valor de 2006 (R \$ 350,00 ou US\$148,93) $(67,8 \%)$.

A prevalência do relato de queda em idosos no último ano foi de 28\% (IC95\%: 25,5-30,7). Entre os idosos com dor há pelo menos um ano, a prevalência de relato de quedas foi $31,6 \%$ (IC95\%: 26,4-37,5), não diferindo da prevalência de relato de queda dos idosos sem dor, que foi de 26,4\% (IC95\%: 23,1-30,0; $p=0,145$ ). A maioria dos idosos com dor há um ano ou mais $(58,9 \%)$ havia caído uma vez nos últimos 12 meses, mas 27,6\% deles relataram três ou mais quedas, e 13,5\%, duas quedas.

Consultando-se a Tabela 1, nota-se que não houve associação entre as variáveis sociodemográficas e relato de queda.

A Tabela 2 apresenta a relação entre as características da dor e a prevalência de relato de queda. É possível observar-se que os idosos com dor há mais de dois anos tiveram maior risco de queda quando comparados com os idosos com dor de um a dois anos.

Na Tabela 3 é apresentada a relação entre queda e morbidade autorreferida. Foram observadas associações entre queda nos últimos 12 meses e as morbidades autorreferidas: hipertensão, artrite/artrose/reumatismo, osteoporose, incontinência urinária, problema nervoso ou psiquiátrico e catarata. 
Tabela 1

Prevalência do relato de queda em idosos com dor de duração a partir de um ano, segundo características sociodemográficas. Projeto SABE, São Paulo, Brasil, 2006 *.

\begin{tabular}{|c|c|c|c|c|}
\hline Variáveis & Prevalência & RP & IC95\% & Valor de $p$ \\
\hline Faixa etária (anos) & & & & 0,111 \\
\hline $60-64$ & 8,1 & 1,00 & & \\
\hline $65-74$ & 15,2 & 1,46 & $0,92-2,33$ & \\
\hline$\geq 75$ & 8,4 & 1,61 & $1,03-2,51$ & \\
\hline Sexo & & & & 0,114 \\
\hline Masculino & 26,2 & 1,00 & & \\
\hline Feminino & 34,5 & 1,32 & $0,93-1,87$ & \\
\hline Trabalha & & & & 0,734 \\
\hline Não & 32,7 & 1,00 & & \\
\hline Sim & 29,8 & 1,07 & $0,74-1,54$ & \\
\hline Vive sozinho & & & & 0,209 \\
\hline Não & 30,3 & 1,00 & & \\
\hline Sim & 40,2 & 0,79 & $0,55-1,14$ & \\
\hline Renda familiar mensal (salários mínimos) ** & & & & 0,256 \\
\hline$\leq 1$ & 45,2 & 1,00 & & \\
\hline$>1 \mathrm{e}<3$ & 28,8 & 0,77 & $0,55-1,08$ & \\
\hline$\geq 3$ & 28,5 & 0,77 & $0,51-1,15$ & \\
\hline
\end{tabular}

IC95\%: intervalo de 95\% de confiança; RP: razão de prevalência.

* $\mathrm{n}=1.169$, representando 895.762 pessoas idosas;

** Salário mínimo: R\$350,00 (valor de referência no ano de 2006, que corresponde a US\$148,93). Valor do dólar em janeiro de $2006=\mathrm{R} \$ 2,35$.

Tabela 2

Prevalência do relato de queda em idosos com dor há pelo menos um ano, segundo características da dor que mais incomodava. Projeto SABE, São Paulo, Brasil, 2006 *

\begin{tabular}{|c|c|c|c|c|}
\hline Variáveis & Prevalência & $\mathrm{RP}$ & IC95\% & Valor de $p$ \\
\hline Tempo com dor (anos) & & & & 0,015 \\
\hline $1-2$ & 28,2 & 1,00 & & \\
\hline$>2$ & 32,2 & 1,39 & $1,06-1,81$ & \\
\hline Local de dor & & & & 0,570 \\
\hline Região de membros inferiores (MMII) & 23,2 & 1,00 & & \\
\hline Região lombar (abaixo da cintura) & 28,5 & 1,22 & $0,80-1,86$ & \\
\hline Outras regiões & 37,5 & 1,21 & $0,84-1,73$ & \\
\hline Intensidade & & & & 0,079 \\
\hline Forte/Intensa/Muito forte/Muito intensa & 38,1 & 1,00 & & \\
\hline Fraca/Média/Moderada & 25,7 & 1,30 & $0,97-1,75$ & \\
\hline Frequência dos episódios & & & & 0,631 \\
\hline Quase todos os dias & 28,5 & 1,00 & & \\
\hline 1 ou 2 vezes por semana & 33,0 & 1,14 & $0,80-1,63$ & \\
\hline 1 vez a cada 15 dias & 30,9 & 1,20 & $0,78-1,86$ & \\
\hline 1 vez por mês & 37,5 & 1,24 & $0,86-1,78$ & \\
\hline
\end{tabular}

IC95\%: intervalo de 95\% de confiança; RP: razão de prevalência.

* $n=340$, representando 260.784 pessoas idosas. 
Prevalência do relato de queda em idosos com dor há pelo menos um ano, segundo morbidades autorreferidas por idosos. São Paulo, Brasil, 2006 *.

\begin{tabular}{|c|c|c|c|c|}
\hline Variáveis & Prevalência & RP & IC95\% & Valor de $p$ \\
\hline Sintomas depressivos & & & & 0,708 \\
\hline Não & 30,5 & 1,00 & & \\
\hline Sim & 31,9 & 1,07 & $0,75-1,53$ & \\
\hline Hipertensão & & & & 0,011 \\
\hline Não & 23,3 & 1,00 & & \\
\hline Sim & 35,7 & 1,62 & $1,12-2,36$ & \\
\hline Diabetes & & & & 0,379 \\
\hline Não & 30,2 & 1,00 & & \\
\hline Sim & 37,5 & 1,17 & $0,83-1,65$ & \\
\hline Asma/Bronquite/Enfisema & & & & 0,719 \\
\hline Não & 31,5 & 1,00 & & \\
\hline Sim & 32,1 & 0,92 & $0,60-1,42$ & \\
\hline Problemas cardíacos & & & & 0,331 \\
\hline Não & 29,3 & 1,00 & & \\
\hline Sim & 34,4 & 1,17 & $0,85-1,62$ & \\
\hline Embolia/Derrame/Ataque/Isquemia/Trombose cerebral & & & & 0,209 \\
\hline Não & 31,0 & 1,00 & & \\
\hline Sim & 39,5 & 1,32 & $0,85-2,05$ & \\
\hline Artrite/Reumatismo/Artrose & & & & 0,007 \\
\hline Não & 27,3 & 1,00 & & \\
\hline Sim & 37,6 & 1,52 & $1,12-2,05$ & \\
\hline Osteoporose & & & & $<0,0001$ \\
\hline Não & 25,6 & 1,00 & & \\
\hline Sim & 46,4 & 1,83 & $1,37-2,45$ & \\
\hline Incontinência urinária & & & & 0,001 \\
\hline Não & 26,8 & 1,00 & & \\
\hline Sim & 41,0 & 1,63 & $1,21-2,19$ & \\
\hline Incontinência fecal & & & & 0,174 \\
\hline Não & 31,3 & 1,00 & & \\
\hline Sim & 35,7 & 1,34 & $0,88-2,05$ & \\
\hline Problema nervoso ou psiquiátrico & & & & 0,003 \\
\hline Não & 29,3 & 1,00 & & \\
\hline Sim & 43,2 & 1,61 & $1,17-2,21$ & \\
\hline Catarata & & & & 0,001 \\
\hline Não & 26,0 & 1,00 & & \\
\hline Sim & 43,1 & 1,62 & $1,20-2,19$ & \\
\hline
\end{tabular}

IC95\%: intervalo de 95\% de confiança; RP: razão de prevalência.

${ }^{\star} \mathrm{n}=340$, representando 260.784 pessoas idosas.

Na Tabela 4, apresentam-se as relações entre queda e grau de dependência, mobilidade e interferência da dor no trabalho, não tendo sido observadas associações. No entanto, houve maior risco de queda entre os idosos com dor que referiram interferência moderada $(\mathrm{p}=0,039)$ ou extrema $(p=0,042)$ da dor no trabalho.

No intuito de identificar as variáveis independentemente associadas à ocorrência de que- da em idosos com dor, deu-se continuidade à análise com a regressão múltipla de Cox (com variância robusta). Utilizaram-se todas as variáveis que apresentaram valores de $\mathrm{p}$ menores que 0,25 na análise univariada: osteoporose, incontinência urinária, catarata, problema nervoso ou psiquiátrico, artrite/reumatismo/artrose, hipertensão, faixa etária, interferência da dor no trabalho normal, sexo, alteração de mobilidade, 
Prevalência do relato de queda em idosos com dor há pelo menos um ano, segundo o grau de dependência, a mobilidade e o domínio de interferência da dor da escala SF 12. São Paulo, Brasil, 2006 *.

\begin{tabular}{|c|c|c|c|c|}
\hline Variáveis & Prevalência & RP & IC95\% & Valor de $p$ \\
\hline Dependência nas ABVD & & & & 0,155 \\
\hline Idosos independentes & 30,6 & 1,00 & & \\
\hline Idosos dependentes & 33,7 & 1,24 & $0,92-1,68$ & \\
\hline Dependência nas AIVDS & & & & 0,156 \\
\hline Idosos independentes & 28,4 & 1,00 & & \\
\hline Idosos dependentes & 33,6 & 1,28 & $0,91-1,78$ & \\
\hline Mobilidade alterada & & & & 0,127 \\
\hline Não & 22,7 & 1,00 & & \\
\hline Sim & 35,2 & 1,37 & $0,91-2,04$ & \\
\hline Interferência da dor no trabalho normal & & & & 0,145 \\
\hline De maneira alguma & 26,5 & 1,00 & & \\
\hline Um pouco & 32,2 & 1,34 & $0,86-2,08$ & 0,199 \\
\hline Moderadamente & 36,9 & 1,56 & $1,02-2,39$ & 0,039 \\
\hline Bastante/Extremamente & 34,5 & 1,56 & $1,01-2,04$ & 0,042 \\
\hline
\end{tabular}

ABVD: atividades básicas de vida diária; AIVD: atividades instrumentais de vida diária; IC95\%: intervalo de $95 \%$ de confiança; RP: razão de prevalência.

${ }^{\star} \mathrm{n}=340$, representando 260.784 pessoas idosas.

renda familiar, dependência para atividades básicas de vida diária, dependência para atividades instrumentais de vida diária, incontinência fecal, intensidade da dor e duração da dor. As variáveis sexo e faixa etária foram incluídas ao final do modelo, para o ajuste por estas variáveis.

A Tabela 5 apresenta o modelo de regressão múltipla para queda e diversas variáveis. Observa-se que a chance de autorrelato de queda para um idoso com dor há pelo menos um ano e com osteoporose foi $50 \%$ maior do que para os sem osteoporose ( $\mathrm{p}=0,019)$. O mesmo aconteceu para os idosos com dor e com incontinência urinária: a chance de queda foi $48 \%$ maior do que para os sem incontinência $(p=0,010)$. A associação de catarata apresentou valores limítrofes com $\mathrm{RP}=$ 1,37 (IC95\%: 0,98-1,92), mas valor de $\mathrm{p}=0,063$. A associação de dor há pelo menos um ano e artrite foi mantida no modelo final, pois, apesar de não ter apresentado valor de $p<0,05(p=0,26)$, ela modificou as razões de prevalência de outras variáveis analisadas (osteoporose e incontinência urinária). Todas as outras variáveis incluídas nas análises perderam significância estatística.

\section{Discussão}

A prevalência de queda foi de $28 \%$ entre os idosos incluídos nas análises, 26,4\% para aqueles sem dor crônica e 31,6\% para os com dor crônica, sem diferença estatística. Alguns estudos não observaram relação entre dor e quedas, à semelhança do observado na presente pesquisa.

Em revisão sistemática que incluiu 74 estudos prospectivos e teve como critérios $80 \%$ ou mais da população residente na comunidade com idade igual ou superior a 65 anos, foram identificados 31 fatores de risco incluídos em, no mínimo, cinco estudos, mas, na metanálise, não se observou relação entre dor e queda 11. Essa revisão incluiu estudos com populações semelhantes à da presente pesquisa, e ambas apresentaram resultados similares.

Estudos brasileiros com idosos residentes na comunidade observaram prevalência de queda entre $27,6 \%$ e $34,8 \%$, similar à encontrada em nossas análises 1,5,14. Estudo transversal que envolveu 4.003 idosos, com 65 anos ou mais, residentes em 41 municípios de sete estados brasileiros, encontrou prevalência de queda de $34,8 \%$, a maior entre mulheres $(40 \%)^{5}$. Siqueira et al. ${ }^{14}$ analisaram a ocorrência de queda no mês anterior à entrevista em 6.616 idosos residentes em cem municípios de 23 estados brasileiros. A prevalência foi de 27,6\% (IC95\%: 26,5-28,7), semelhante à observada na presente pesquisa. Perracini \& Ramos 1 realizaram um seguimento de dois anos, por meio de inquérito domiciliar, com 1.667 idosos com 65 anos ou mais, todos 
Estimativas da razão de prevalência do autorrelato de queda feito por idosos com dor há pelo menos um ano, com base no modelo de regressão múltipla de Cox. São Paulo, Brasil, 2006.

\begin{tabular}{|c|c|c|c|}
\hline Variáveis & $\mathrm{RP}_{\mathrm{br}}$ & $\mathrm{RP}_{\mathrm{aj}}(\mathrm{IC} 95 \%)$ & Valor de $p$ \\
\hline Osteoporose & & & 0,019 \\
\hline Não & 1,00 & & \\
\hline Sim & 1,83 & $1,50(1,06-2,10)$ & \\
\hline Incontinência urinária & & & 0,010 \\
\hline Não & 1,00 & & \\
\hline Sim & 1,63 & $1,48(1,09-2,01)$ & \\
\hline Catarata & & & 0,063 \\
\hline Não & 1,00 & & \\
\hline Sim & 1,62 & $1,37(0,98-1,92)$ & \\
\hline \multicolumn{4}{|l|}{ Artrite } \\
\hline Não & 1,00 & & 0,25 \\
\hline Sim & 1,52 & $1,21(0,87-1,71)$ & \\
\hline
\end{tabular}

$\mathrm{RP}_{\mathrm{br}}$ : razão de prevalência bruta; $\mathrm{RP}_{\mathrm{aj}}$ : razão de prevalência ajustada para sexo e faixa etária.

residentes na cidade de São Paulo, e encontraram que a prevalência de quedas, no último ano, foi de $31 \%$, também semelhante à observada nesta investigação.

Não se observou associação entre queda e variáveis sociodemográficas (Tabela 1), podendo-se levantar algumas hipóteses. Quanto à idade, nota-se que grande parte dos idosos tinha entre 60 e 74 anos, o que aponta para uma população mais homogênea, talvez com as mesmas condições de funcionalidade. A idade se associou à queda nos estudos de Gama \& GómezConesa 7, Siqueira et al. 5 , Siqueira et al. 14 , Deandrea et al. 15, indicando que os "muito idosos" (acima de 75 ) caem mais que os idosos.

Estudos internacionais com idosos da comunidade vivenciando dor crônica observaram prevalência de dor que variou entre $25,3 \%$ e 57,9\%, conforme o local e a natureza da dor; é possível que, em muitos estudos, tenham sido incluídas dores agudas e crônicas. Entre idosos com fibromialgia (múltiplos locais de dor), a prevalência de queda foi de $40 \% 16$; entre aqueles com dor no quadril, foi de $45 \% 15$; idosos com dor no pé apresentaram prevalência de 57,9\% 17 e, entre idosos com queixa de dor em geral, relato de queda ocorreu entre $25,3 \%$ dos avaliados 18 . Nota-se que alguns dos valores descritos são superiores à prevalência observada na presente pesquisa $(28 \%$ sendo, $26,4 \%$ para os sem dor crônica e $31,6 \%$ para os com dor crônica, sem diferença estatística).

Neste estudo, os aspectos que demonstraram associação com queda foram os seguintes: dor por mais de dois anos, o que aumentou o risco de queda em 39\% ( $p=0,015$ ) em comparação com ausência de dor; dor que interferia moderadamente e bastante no trabalho, aumentando em $56 \%$ a chance de ter queda $(p=0,039$ e $p=0,042$, respectivamente). No entanto, essas associações perderam significância nas análises múltiplas. Não foram encontrados estudos que avaliassem a duração da dor e sua associação com queda.

Quanto à interferência moderada da dor no trabalho, encontrou-se um estudo que observou resultados semelhantes aos encontrados na análise univariada. Pesquisa longitudinal com 749 idosos acima de setenta anos demonstrou que aqueles cujo relato, na primeira coleta, foi de "dor que interferia nas atividades diárias" tiveram maior risco de queda 12 .

As características e os locais de dor que aumentaram a ocorrência de queda foram queixa de dor em geral 10,19 , dor no pé $3,11,17$, dor no quadril 20 , dor em dois ou mais locais 21 , dor em região dorsal e cervical 22 , dor em duas ou mais articulações 12, dor em locais diferentes de membros inferiores 3 , dor generalizada de moderada a intensa 3 , dores mais intensas 12 e dores que interferem nas atividades diárias 4,12.

Nos diversos estudos sobre fatores de risco para quedas entre idosos, limitações funcionais estão frequentemente associadas a esse evento 1,7,14,15, entretanto, na presente investigação, não se observou associação entre funcionalidade e queda. A explicação para isso pode ser o fato de que idosos, mesmo sem limitações funcionais, são portadores de outras doenças que os predispõem a quedas. Outro fato que pode explicar 
nossos achados é que, apesar de dor e maior limitação funcional terem se associado 23 , houve considerável limitação funcional entre os idosos sem dor, o que pode ter levado a risco de queda igual para os dois grupos.

Outro aspecto que pode explicar a não ocorrência de associação entre funcionalidade e queda está relacionado às escalas utilizadas para avaliação da funcionalidade. Nesta pesquisa, foram utilizadas, nas análises, as escalas de atividades básicas e instrumentais e de mobilidade, porém todas baseadas no autorrelato do idoso sobre sua capacidade funcional. Por haver uma considerável diversidade de escalas validadas e usadas nos estudos, é difícil estabelecerem-se comparações. Pesquisas com idosos brasileiros as quais utilizaram outras escalas na avaliação funcional e atividade física e não incluíram dor nas análises encontraram associação entre maior dependência, sedentarismo e quedas 1,5,14.

Vale ressaltar que os fatores de risco para queda são diversos e que as estratégias empregadas para avaliar a ocorrência são também muito variadas, o que dificulta a comparação entre achados.

Estudos de revisão e metanálise tentaram definir quais características causariam maior risco aos idosos 7,15 e encontraram osteoporose, incontinência urinária e artrite como fatores de risco para quedas. Esse achado está de acordo com os resultados desta pesquisa, na qual se observou que, entre os idosos com dor, artrite influiu na prevalência de queda, enquanto osteoporose e incontinência apresentaram-se como fatores de risco.

Fato que pode dificultar os estudos envolvendo osteoporose é o baixo índice de diagnóstico dessa patologia, observado na prática e comprovado em pesquisa. Vários estudos tentam explicar a relação entre osteoporose e quedas, como a alteração postural provocada pela doença, que resulta em aumento da cifose cervical e torácica, mudança do centro de pressão e equilíbrio. Contudo, alguns mecanismos compensatórios poderiam diminuir o risco de queda, de modo que os resultados ainda são inconclusivos $24 . \mathrm{Na}$ literatura internacional, é frequente a ênfase em se prevenir quedas em idosos com osteoporose visando à prevenção de fraturas e incapacidades.

Comumente, alterações radiológicas de artrite não se constituem em risco para queda, o que foi comprovado em dois estudos 25. Observou-se que os sintomas e as alterações provocados pela artrite influenciam no risco de quedas entre idosos, mais do que somente alterações radiológicas. Um destes sintomas é a dor. Assim, o fato que parece explicar a relação entre artrite e quedas é a ocorrência de sintomatologia álgica 25 .
A literatura não é conclusiva sobre incontinência urinária e queda, mas alguns achados brasileiros e internacionais indicaram-na como fator de risco para quedas entre idosos 18,26,27,28,29.

Estudo caso-controle, realizado no Rio de Janeiro, comparou 250 idosos internados com fratura de fêmur em hospitais públicos com 250 idosos da comunidade, pareados por sexo, idade e local de moradia. Os fatores que aumentaram o risco de quedas foram baixo índice de massa corporal (IMC), déficit cognitivo, história de acidente vascular encefálico (AVE), incontinência urinária (OR = 3,05; IC95\%: 1,82-5,12; p < 0,001), uso de benzodiazepínicos e de relaxantes musculares 26. Em nossos achados, incontinência urinária também foi fator de risco para queda; por outro lado, embora o uso de relaxantes musculares possa indicar a existência de dor, esta não apareceu como fator de risco.

Em estudo de metanálise, incontinência urinária mostrou-se fator de risco para quedas, com odds ratio $(\mathrm{OR})$ variando de 1,33 , para quedas em geral, a 1,71, para quedas recorrentes, e p < 0,005 15. Uma hipótese para a associação entre dor, incontinência e queda entre idosos é a de que o medo de perder urina resulte em deslocamentos mais bruscos, rápidos e menos cuidadosos, aumentando o risco de quedas. As causas de tal associação ainda não são claras.

Análise de dados do projeto SABE demonstrou que, em São Paulo, ser do sexo feminino, ter idade mais avançada, apresentar maior escore de sintomas depressivos e diabetes foram fatores de risco para queda 22 . No projeto, a dor não aparece como provável fator de risco, pois os dados analisados referem-se à coleta do ano 2000, quando as questões sobre dor ainda não haviam sido incluídas.

Do descrito, nota-se que as três variáveis que se mantiveram no modelo final são também reconhecidas em outras pesquisas como fatores de risco para quedas em idosos: osteoporose 24,28,30, incontinência urinária 7,15,18,22,25,26,27 e artrite $7,9,25,26$. Todavia, há estudos nos quais incontinência urinária 1,15,29, artrite 31 e osteoporose 26,29,32 não apresentaram relação com queda.

Não há uma proposta internacional de padronização dos fatores de risco e das formas de avaliação destes nos estudos sobre quedas; dessa forma, variáveis significativas em alguns estudos não são incluídas em outros. Assim, a literatura apresenta considerável número de fatores de risco, e alguns deles, como sexo feminino, maior faixa etária, história anterior de queda, alterações de mobilidade e maior dependência, são mundialmente reconhecidos como fatores de risco para queda. 
Diante dos resultados aqui apresentados, há que se reforçar a importância de ações de prevenção de quedas em idosos. As consequências das quedas na qualidade de vida e sobrevida de idosos são comprovadas, logo a implementação de programas que visem à prevenção deveria ser prioridade do governo. A multicausalidade desse fenômeno reforça a importância de ações intersetoriais na sociedade. Culpar os tapetes nos domicílios dos idosos é uma visão simplista da dimensão das quedas, que devem ser enfrentadas como um problema de saúde pública. Além disso, o conhecimento científico sobre o tema, que inclui revisões sistemáticas 7,11,33, aponta claramente os fatores de risco e as respectivas intervenções com evidências científicas que devem respaldar as propostas de ações nos serviços de saúde e em toda a sociedade.

A inclusão do tema dor no projeto SABE 2006 viabilizou a determinação da prevalência e características da dor crônica em idosos da comunidade e sua associação com quedas, dados raros ou inexistentes na literatura nacional, contribuindo dessa forma para o entendimento desses agravos. Por se tratar de uma análise transversal, nenhuma relação de causalidade pôde ser inferida.

Outro aspecto a considerar é o fato de que os dados analisados se baseiam exclusivamente no autorrelato dos idosos sobre quedas. São ampla- mente difundidas as potencialidades e debilidades dessa estratégia de coleta de dados; entre as primeiras, há fortes indícios de que a memória seja eficaz para fatos marcantes, como a queda. Vale ressaltar, como aspecto positivo, que o projeto SABE realizou criteriosa avaliação do estado cognitivo do idoso e utilizou informante substituto nos casos de déficit cognitivo importante, visando a se obter maior confiabilidade das informações coletadas.

A dor não tem sido incluída frequentemente como variável nas pesquisas, o que dificulta concluir se o risco de quedas é aumentado por esse agravo; é também difícil apontar quais características da dor ampliam esse risco. As pesquisas que abordam a dor e sua associação com queda não são suficientes para responder a essas questões. Estudos de coorte com número representativo de idosos que vivem na comunidade poderiam trazer contribuições nesse sentido, esclarecendo relações como local de dor e queda, além de intensidade e duração da dor e queda.

Embora queda seja um evento multideterminado, o conhecimento atual indica que um conjunto de fatores leva à ocorrência desse agravo. Muitos deles são passíveis de controle, como osteoporose e incontinência urinária, conforme observado no presente estudo.

\section{Resumen}

El objetivo de este estudio fue evaluar la asociación entre dolor crónico y el auto-reporte de las caídas. Estudio transversal, de base poblacional de personas de edad avanzada sin déficit cognitivo, residentes en la ciudad de São Paulo, Brasil. Los ancianos con dolor crónico lo definen como un dolor prolongado durante hace más de un año. Los datos fueron recogidos mediante entrevistas y los análisis se realizaron utilizando Stata 11.0. La prevalencia de dolor crónico fue de 29,7 \% (IC95\%: 25,4-33,9), la prevalencia de caídas en el último año, entre las personas de edad avanzada con dolor fue de 31,6\% (IC95\%: 26,4-37,5) y no difieren de las personas de edad avanzada sin dolor (26,4\%; IC95\%: 23,1-30,0; $p=0,145$ ). Sin embargo, cuando existía dolor y se sufría osteoporosis o existía dolor e incontinencia urinaria, el riesgo de caídas fue de $50 \%(p=0,019)$ y 48\% $(p=0,010)$, respectivamente. La asociación dolor crónico y la osteoporosis o incontinencia urinaria aumenta la posibilidad de caídas en el anciano.

\section{Colaboradores}

M. S. G. Dellaroza foi responsável pela organização e elaboração do artigo na íntegra, além das correções finais para publicação. C. A. M. Pimenta contribuiu para a realização da pesquisa desde a criação; revisou o texto do artigo, com contribuições em sua redação final. M. L. Lebrão contribuiu com a operacionalização da pesquisa e com a revisão final do texto do artigo. Y. A. O. Duarte revisou do texto final. P. E. Braga revisou a apresentação de dados na seção de resultados.

\section{Agradecimentos}

O estudo SABE obteve apoio financeiro da Fundação de Amparo à Pesquisa do Estado de São Paulo para a fase de coleta 2006. Recursos complementares foram obtidos junto ao CNPq (Edital MCT/CNPq 14/2008 - Universal, por meio do Projeto Dor Crônica e Incapacidade, número 470856/2008-4). 
1. Perracini MR, Ramos LR. Fatores associados a que das em uma coorte de idosos residentes na comunidade. Rev Saúde Pública 2002; 36:709-16.

2. Ribeiro AP, Souza ER, Atie S, Souza AC, Schilithz AO. A influência das quedas na qualidade de vida de idosos. Ciênc Saúde Coletiva 2008; 13:1265-73.

3. Leveille SG, Bean J, Bandeen Roche K, Jones R, Hochberg M, Guralnik JM. Musculoskeletal pain and risk for falls in older disabled women living in the community. J Am Geriatr Soc 2002; 50:671-8.

4. Blyth FM, Cumming R, Mitchell P, Wang JJ. Pain and falls in older people. Eur J Pain 2007; 11:56471 .

5. Siqueira FV, Fachini LA, Silveira DS, Piccini RX, Tomasi E, Thumé E, et al. Prevalência de quedas em idosos e fatores associados. Rev Saúde Pública 2007; 41:749-56.

6. Gawryszewski VP. A importância das quedas no mesmo nível entre idosos no estado de São Paulo. Rev Assoc Med Bras 2010; 56:162-7.

7. Gama ZAS, Gómez-Conesa A. Factores de riesgo de caídas en ancianos: revisión sistemática. Rev Saúde Pública 2008; 42:946-56.

8. Pimenta CAM, Cruz DALM, Rosseto EG, Krelling MCGD, Dellaroza MSG. Epidemiologia da dor. In: Figueiró JAB, Angelotti G, Pimenta CAM, organizadores. Dor e saúde mental. São Paulo: Editora Atheneu; 2005. p. 3-22.

9. Dellaroza MSG, Pimenta CAM, Matsuo T. Prevalência e caracterização da dor crônica em idosos não institucionalizados. Cad Saúde Pública 2007; 23:1151-60.

10. Bishop MD, Meuleman J, Robinson M, Light KE. Influence of pain and depression on fear of falling, mobility, and balance in older male veterans. J Rehabil Res Dev 2007; 44:675-83.

11. Chaiwanichsiri D, Janchai S, Tantisiriwat N. Foot disorders and falls in older persons. Gerontology 2009; 55:296-302.

12. Leveille SG, Jones RN, Kiely DK, Hansdorlf JM, Shmerling RH, Guralnik JM, et al. Chronic musculoskeletal pain and the occurrence of falls in an older population. JAMA 2009; 302:2214-21.

13. Guimarães JMN, Farinatti PTV. Análise descritiva de variáveis teoricamente associadas ao risco de quedas em mulheres idosas. Rev Bras Med Esporte 2005; 11:299-305.

14. Siqueira FV, Fachini LA, Silveira DS, Piccini RX, Tomasi E, Thumé E, et al. Prevalence of falls in elderly in Brazil: a countrywide analysis. Cad Saúde Pública 2011; 27:1819-26.

15. Deandrea S, Lucenteforte E, Bravi F, Foschi R, Vecchia C, Negri E. Risk factors for falls in community-dwelling older people a systematic review and meta-analysis. Epidemiology 2010; 21:658-68.

16. Rutledge DN, Cherry BJ, Rose DJ, Rakovski C, Jones CJ. Do fall predictors in middle aged and older adults predict fall status in persons 50+ with fibromyalgia? An exploratory study. Res Nurs Health 2010; 33:192-206.

17. Mickle KJ, Munro BJ, Lord SR, Menz HB, Steele JR. Foot pain, plantar pressures, and falls in older people: a prospective study. J Am Geriatr Soc 2010; 58:1936-40.
18. Yagci N, Cavlak U, Aslan UB, Akdag B. Relationship between balance performace and musculoskeletal pain in lower body comparison healthy middle aged and older adults. Arch Gerontol Geriatr 2007; 45:109-19.

19. Divani AA, Vazquez G, Barret AM, Asadollahi M, Luft AR. Risk factors associated with injury attributable to falling among elderly population with history of stroke. Stroke 2009; 40:3286-92.

20. Arnold CM, Faulkner RA. The history of falls and the association of the timed up and go test to falls and near-falls in older adults with hip osteoarthritis. BMC Geriatrics 2007; 7:17.

21. Leveille SG, Kiel DP, Jones RN, Roman A, Hannan MT, Sorond FA, et al. The Mobilize Boston study: design and methods of a prospective cohort study of novel risk factors for falls in an older population. BMC Geriatrcs 2008; 18:8-16.

22. Reyes-Ortiz CA, Al Snish S, Markides KS. Falls among elderly persons in Latin American and the Caribbean and among elderly Mexican-Americans. Rev Panam Salud Pública 2005; 17:362-9.

23. Dellaroza MSG, Pimenta CAM, Duarte YA, Lebrão ML. Dor crônica em idosos residentes em São Paulo, Brasil: prevalência, características e associação com capacidade funcional e mobilidade (Estudo SABE). Cad Saúde Pública 2013; 29:325-34.

24. Sinaki M, Brey RH, Hughes CA, Larson DR, Kaufman KR. Balance disorder and increased risk of falls in osteoporosis and kyphosis: significance or kyphotic posture and muscle strength. Osteoporos Int 2005; 16:1004-10.

25. Foley SJ, Lord SR, Srikanth V, Cooley H, Jones G. Falls risk is associated with pain and dysfunction but not radiographic osteoarthritis in older adults: Tasmanian older adult cohort study. Osteoarthritis Cartilage 2006; 14:533-9.

26. Coutinho ESFS, Dutra S. Uso de medicamentos como fator de risco para fratura grave decorrente de queda em idosos. Cad Saúde Pública 2002; 18:1359-66.

27. Moreira MD, Costa AR, Caldas CP. The association between nursing diagnoses and the occurrence of falls observed among eldery individuals assisted in an outpatient facility. Rev Latinoam Enferm 2007; 15:311-7.

28. Nieuwenhuizen RC, Dijk N, Breda FG, Scheffer AC, Korevaar JC, Cammen TJ, et al. Assessing the prevalence of modifiable risk factors in older patients visiting an ED due to a fall using the CAREFALL Triage Instrument. Am J Emerg Med 2010; 28:9941001.

29. Halil M, Ulger Z, Cankurtaran M, Shorbagi A, Yavuz BB, Dede D, et al. Falls and the elderly: is there any difference in the developing world? A crosssectional study from Turkey. Arch Gerontol Geriatrics 2006: 43:351-9.

30. Silva RB, Costa-Paiva L, Oshima MM, Morais SS, Pinto-Neto AM. Frequência de quedas e associação com parâmetros estabilométricos de equilíbrio em mulheres na pós-menopausa com e sem osteoporose. Rev Bras Ginecol Obstet 2009; 31:496-502. 
31. Andresen EM, Wolinsky FD, Miller P, Wilson MMG, Malmstrom TK, Miller DK. Cross sectional and longitudinal risk factors for falls, fear of falling, and falls efficacy in a cohort of middle aged african Americans. Gerontologist 2006; 46:249-57.

32. Pinheiro MP, Ciconelli RM, Martini LA, Ferraz MB. Risk factors for recurrent falls among Brazilian women and men: the Brazilian Osteoporosis Study (BRAZOS). Cad Saúde Pública 2010; 26:89-96.
33. Gillespie LD, Gillespie WJ, Robertson MC, Lamb SE, Cumming RG, Rowe BH. Interventions for preventing falls in elderly people. Cochrane Database Syst Rev 2003; (4):CD000340.

Recebido em 16/Nov/2012

Versão final reapresentada em 19/Jun/2013

Aprovado em 17/Set/2013 